\title{
Hyperparathyroidism, arterial hypertension and aortic stiffness: a possible bidirectional link between the adrenal cortex and the parathyroid glands that causes vascular damage?
}

\author{
Gian Paolo Rossi \\ Hypertension Research (2011) 34, 286-288; doi:10.1038/hr.2010.251; published online 16 December 2010
}

$\mathrm{P}$ rimary hyperparathyroidism $(\mathrm{PPTH})$ is generally thought to be a common endocrine disorder. However, it represents an uncommon endocrine cause of arterial hypertension, with a likely prevalence among hypertensive patients of less than $0.01 \%$ an imprecise estimate because of the lack of prospective studies.

However, between $56 \%$ and $80 \%$ of the patients with PPTH have high blood pressure (BP) and display hypertension-related target organ damage. On the basis of large studies from Scandinavia, a high rate of cardiovascular complications and mortality that has been consistently observed in PPTH patients compared with patients with essential hypertension without PPTH; this can be related to an excess organ damage in PPTH patients. ${ }^{1}$

Despite these findings, the mechanisms by which PPTH is associated with hypertension and increased cardiovascular risk remain poorly understood and somewhat intriguing. In fact, parathyroid hormone (PTH) exerts a vasodilatory effect in different models ${ }^{2,3}$ and induces an endothelium-dependent vasodilatory effect through increased nitric oxide production, ${ }^{4,5}$ both of which are effects that would be expected to decrease, rather than increase, BP. However, PPTH has also been associated with endothelial dysfunction, which is usually due to reduced nitric oxide

Professor GP Rossi is at the DMCS_Clinica Medica 4, Policlinico Universitario, Via Giustiniani 2, 35126 Padova, Italy.

E-mail: gianpaolorossi@unipd.it bioactivity, and represents a hallmark of high BP and many other conditions that are associated with increased cardiovascular risk. In contrast with this finding, increased levels of markers that are downstream of nitric oxide signaling, which were normalized after parathyroidectomy, have also been documented in PPTH patients. ${ }^{6}$ Therefore, it has been proposed that increased nitric oxide bioactivity is a compensatory mechanism that counteracts the pressor effects of PTH-induced increases in intracellular calcium. ${ }^{6}$

In this issue of the journal, Rosa et al. ${ }^{7}$ report on their investigation of PPTH patients based on measurements of pulse wave velocity (PWV) before and after surgical treatment of PPTH; PWV is an index of aortic stiffness and hypertension-related target organ damage, which is recommended by the current guidelines of the ESC/ESH as part of the standard evaluation of hypertensive patients because it conveys information that is useful for risk stratification purposes. Interestingly, they found that PWV was higher in PPTH patients than in essential hypertensive patients, which suggests that excess PTH changes the mechanical properties of the arterial walls. However, this important finding is not consistent with the results of another recent study from Israel: compared with control subjects who were matched for age, gender and CV risk factors, neither the patients with full-blown (hypercalcemic) PPTH nor those with mild (normocalcemic) PPTH showed increased arterial stiffness, as assessed by several indices, including the PWV. ${ }^{8}$ Although these inconsistencies could reflect either a selection bias, involving differences in ethnicity, overall cardiovascular risk profile, stage of PPTH disease in the patients and/or different selection criteria for the controls, it should be emphasized that in the study by Rosa et al., ${ }^{7}$ removal of parathyroid adenoma and correction of PPTH were followed by resolution of the increased PWV and a decrease in BP. Therefore, that excess PTH per se has a major detrimental effect on the arterial wall, as shown by increased BP and induced stiffening of the aorta. The aortic stiffening obviously implies increased afterload and contributes to increasing both systolic and diastolic BP. Notably, somewhat inconsistent with this theory, the authors could not demonstrate any significant relationship between the PWV values and either serum PTH levels or any other index of calcium metabolism, which led them to conclude that the major factor accounting for the postoperative decrease in PWV was the decrease in BP.

Although the results by Rosa et al. are interesting for several reasons, they are, unfortunately, merely observational. Therefore, they do not provide insight into the underlying mechanisms of PTH-induced increases in BP and arterial wall stiffness. In this context, it must be considered that the increase in ionized serum calcium $\left[\mathrm{Ca}^{2+}\right]$, which is a hallmark of overt PPTH, can lead to both increased vascular responsiveness to many vasoconstrictor stimuli and to the activation of signaling events such as TGF $\beta 1$ stimulation, which lead to tissue fibrosis and thus to stiffening of the arterial wall.

Moreover, in vitro data strongly support a relationship between excess PTH and 
the activation of, for example, the reninangiotensin-aldosterone system, one of the major pressor systems. In 2001, on the basis of investigations in human adrenocortical zona glomerulosa cells, our group documented a concentration-dependent secretagogue effect of two peptides on aldosterone: PTH and PTH-related peptide, which is the main mediator of paraneoplastic hypercalcemia. ${ }^{9}$ These findings suggested that chronic hyperparathyroidism and paraneoplastic hypercalcemia can be instrumental in causing and/or maintaining a secretion of aldosterone that is inappropriately high for BP and sodium intake. The ensuing relative hyperaldosteronism can increase BP and cause vascular inflammation, remodeling and fibrosis (Figure 1). This effect is relevant to a much broader population than to patients with $\mathrm{PPTH}$ because an inappropriately high secretion of aldosterone is a feature of primary (essential) hypertension associated with overweight-obesity. ${ }^{10}$ Thus, at least one important pressor mechanism involving aldosterone secretion could account for the occurrence of hypertension and arterial stiffening in the PPTH patients studied by Rosa et al.

Unfortunately, the authors failed to provide data on renin and aldosterone, which precludes the possibility of testing the hypothesis that a correlation exists between
PTH and these hormones. Nonetheless, their findings can have important and broad implications for the vast population of hypertensive patients, particularly those who are overweight or obese.

It is also likely that the functional link between PTH and aldosterone is not confined to PPTH; milder forms of hyperparathyroidism, secondary to a deficiency of $25-\mathrm{OH}$ vitamin $\mathrm{D}$ and/or due to chronic renal insufficiency, are quite common among hypertensive patients. Moreover, when infused into normotensive rats, aldosterone caused an increase in $\mathrm{PTH}$, which was attributed to the decrease in serum $\left[\mathrm{Ca}^{2+}\right]$ due to the hypercalciuria that accompanies the escape phenomenon. ${ }^{11}$ These observations suggest a bidirectional relationship between the adrenocortical zona glomerulosa and the parathyroid gland (Figure 1). Hence, secondary hyperparathyroidism can contribute to the maintenance of a relative degree of hyperaldosteronism, and therefore to increased BP under these common conditions. The hyperaldosteronism could consequently contribute to increases in $\mathrm{PTH}$, thus instituting a feed-forward mechanism.

Given the role of excess aldosterone in causing oxidative stress, this mechanism might also contribute to the activation of inflammatory and fibrogenetic changes.

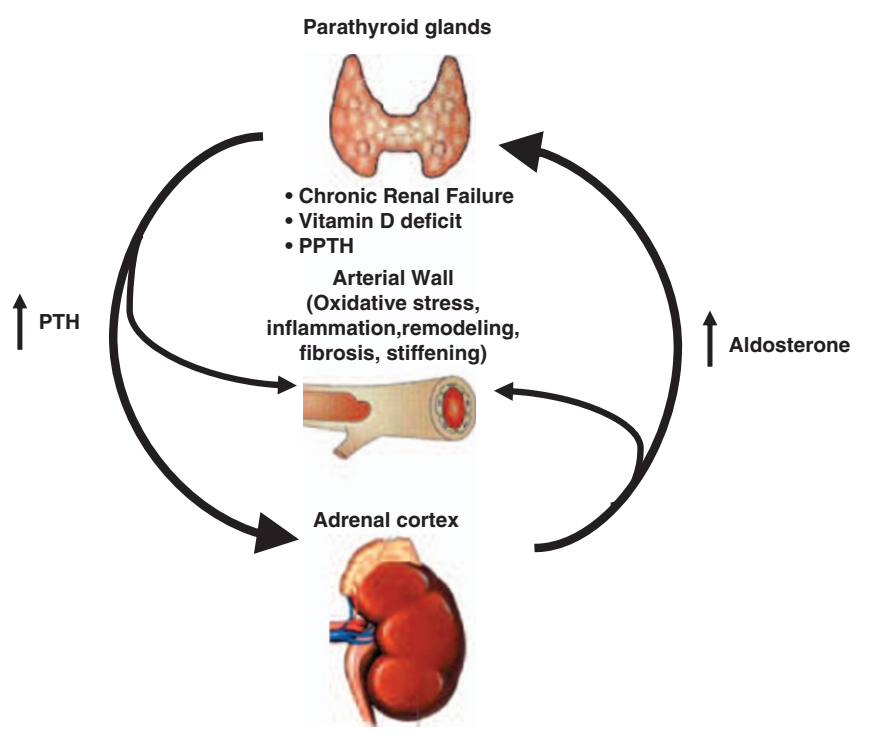

Figure 1 Given the secretagogue effect of parathyroid hormone (PTH) on aldosterone, the excess secretion of PTH can cause absolute or relative hyperaldosteronism; the excess secretion may be either due to primary hyperparathyroidism (PPTH) or secondary to 25-OH vitamin D deficiency or to its impaired renal activation by $1 \alpha$ hydroxylase in patients with chronic renal insufficiency. The absolute or relative hyperaldosteronism, in turn, can contribute to the maintenance of increased PTH secretion, thus creating a feed-forward mechanism. As excess levels of both PTH and aldosterone have a detrimental effect on the vascular wall, the interaction between these hormones can result in vascular damage, which may explain the arterial stiffening described in the paper by Rosa et al. and the excess rate of cardiovascular morbidity and mortality reported in PPTH patients.

Therefore, the functional link between PTH and aldosterone might have an amplification role in inducing the vascular changes that are associated with cardiovascular events (Figure 1).

In summary, the finding that PPTH is associated with increased stiffness of large elastic arteries, in both hypertensive and normotensive patients, along with the observation that aortic PWV is normalized after correction of the PPTH, strongly supports a cause-effect relationship between excess PTH and changes in the mechanical properties of the walls of large elastic arteries. As this result does not appear to be consistent with other recent reports in the literature, further larger prospective studies are necessary. If these studies will eventually confirm the theory that PPTH is associated with stiffening of the large arteries, the underlying mechanisms of these changes need to be carefully dissected.

Moreover, it seems that we are only beginning to appreciate the complex relationship between two major hormonal systems-the calcium-regulating hormones and the reninangiotensin-aldosterone system-that have classically been thought to act independently but, in reality, seem to closely interact. Therefore, further research aimed at clarifying the interaction between the hormones and vitamins that regulate calcium homeostasis and the renin-angiotensin-aldosterone system is necessary. This research is particularly relevant given the increasing prevalence of vitamin $\mathrm{D}$ deficiency and/or chronic renal failure, two conditions that cause secondary hyperparathyroidism, in the aging population, and, therefore, may be related to an increase in systolic BP.

\section{CONFLICT OF INTEREST}

The author declares no conflict of interest.

\section{ACKNOWLEDGEMENTS}

This study was supported by a research grant from the University of Padua, FORICA (The Foundation for Advanced Research in Hypertension and Cardiovascular Diseases) and the Società Italiana dell'Ipertensione Arteriosa.

1 Hedback GM, Oden AS. Cardiovascular disease, hypertension and renal function in primary hyperparathyroidism. J Intern Med 2002; 251: 476-483.

2 Nickols GA, Metz MA, Cline Jr WH. Vasodilation of the rat mesenteric vasculature by parathyroid hormone. J Pharmacol Exp Ther 1986; 236: 419-423.

3 Suzuki Y, Lederis K, Huang M, LeBlanc FE, Rorstad OP. Relaxation of bovine, porcine and human brain arteries by parathyroid hormone. Life Sci 1983; 33: 2497-2503. 
4 Rashid G, Bernheim J, Green J, Benchetrit S. Parathyroid hormone stimulates the endothelial nitric oxide synthase through protein kinase $A$ and $C$ pathways. Nephrol Dial Transplant 2007; 22: 2831-2837.

5 Ohta T, Okamoto E, Shimoya M, Nakazato $\mathrm{Y}$, Ito S. Relaxant mechanisms of parathyroid hormone in rat mesenteric artery. J Cardiovasc Pharmacol 2002; 40: 554-563.

6 Martina V, Bruno GA, Brancaleoni V, Zumpano E, Tagliabue M, Fornengo R, Gasparri G, Pescarmona GP. Calcium blood level modulates endogenous nitric oxide action: effects of parathyroidectomy in patients with hyperparathyroidism. J Endocrinol 1998; 156: 231-235.
7 Rosa J, Raska I Jr, Wichterle D, Petrak O, Strauch B, Somloova Z, Zelinka T, Holaj R, Widimsky J Jr. Pulse wave velocity in primary hyperparathyroidism and effect of surgical therapy. Hypertens Res 2011; 34: 296-300.

8 Tordjman KM, Yaron M, Izkhakov E, Osher E, Shenkerman G, Marcus-Perlman Y, Stern N. Cardiovascular risk factors and arterial rigidity are similar in asymptomatic normocalcemic and hypercalcemic primary hyperparathyroidism. Eur J Endocrinol 2010; 162: 925-933.

9 Mazzocchi G, Aragona F, Malendowicz LK, Nussdorfer GG. PTH and PTH-related peptide enhance steroid secretion from human adrenocortical cells. Am J Physiol Endocrinol Metab 2001; 280: E209-E213.

10 Rossi GP, Belfiore A, Bernini G, Fabris B, Caridi G, Ferri C, Giacchetti G, Letizia C, Maccario M, Mannelli M, Palumbo G, Patalano A, Rizzoni D, Rossi E, Pessina AC, Mantero F. Body mass index predicts plasma aldosterone concentrations in overweight-obese primary hypertensive patients. J Clin Endocrinol Metab 2008; 93 : 2566-2571.

11 Chhokar VS, Sun Y, Bhattacharya SK, Ahokas RA, Myers LK, Xing Z, Smith RA, Gerling IC, Weber KT. Hyperparathyroidism and the calcium paradox of aldosteronism. Circulation 2005; 111: 871-878. 